\title{
Quantum localization of Classical Mechanics
}

\author{
Igor A. Batalin $(a, b) 1$ and Peter M. Lavrov ${ }^{(b, c)} 2$ \\ (a) P.N. Lebedev Physical Institute, \\ Leninsky Prospect 53, 119991 Moscow, Russia \\ (b) Tomsk State Pedagogical University, \\ Kievskaya St. 60, 634061 Tomsk, Russia \\ (c) National Research Tomsk State University, \\ Lenin Av. 36, 634050 Tomsk, Russia
}

\begin{abstract}
Quantum localization of classical mechanics within the BRST-BFV and BV (or fieldantifield) quantization methods are studied. It is shown that a special choice of gauge fixing functions (or BRST-BFV charge) together with the unitary limit leads to Hamiltonian localization in the path integral of the BRST-BFV formalism. In turn, we find that a special choice of gauge fixing functions being proportional to extremals of an initial non-degenerate classical action together with a very special solution of the classical master equation result in Lagrangian localization in the partition function of the BV formalism.
\end{abstract}

Keywords: Classical Mechanics, Hamiltonian BRST-BFV formalism, BV-formalism, Hamiltonian localization, Lagrangian localization

PACS numbers: 11.10.Ef, 11.15.Bt

\footnotetext{
${ }^{1}$ E-mail: batalin@lpi.ru

${ }^{2}$ E-mail: lavrov@tspu.edu.ru
} 


\section{Introduction and Summary}

In Theoretical Physics, one considers usually how to quantize complex dynamical systems, possibly with nontrivial geometry of phase space or constraints of both classes, by observing precisely all the fundamental Physical Principles 1, 2, 3, 4, 5. Although the general quantization problem is of great importance, it is also very interesting to realize what is the status of the classical dynamics from the point of view of an equivalent special quantum theory. In other words, it is interesting to study, how to formulate a version of a quantum theory capable, in a consistent way, to suppress all quantum fluctuations in a given dynamical system, so that the functional path integral reduces to the delta - functional concentrated at the classical trajectory, multiplied by the Jacobian of the argument of the delta - functional with respect to the trajectory. That is exactly what we actually mean when saying about the general quantum localization. There exist a rather numerous series of articles where such a program has been realized and studied [6, 7, 8, 9, 10]. One of the most known approaches is to introduce, in a Hamiltonian dynamics, in addition to the usual Boson time, the two Fermion time components [10]. The linear coefficients of a series expansion of the trajectory in the new Fermion times become then a kind of Hamiltonian ghosts, while the quadratic coefficients become Lagrange multipliers yielding just the delta - functional concentrated at the classical trajectory. In turn, the Hamiltonian ghosts yield the Jacobian required of the delta - functional. Although that approach by itself looks very nice, it seems that the idea of two Fermion extra times is rather artificial. On the other hand, in that scheme, the localization is essentially discrete, in the sense that there is no "parameter" interpolating continuously, as "switching on" quantum fluctuations.

In the present article, we suggest to use the well-known BRST - BFV scheme invented originally for generalized canonical quantization of relativistic dynamical systems with first class constraints [11, 13]. We suggest an unusual form for the BRST - BFV charge and gauge Fermion, and show that there exists such a choice of the gauge - fixing functions that, in the unitary limit, the path integral reproduces exactly the general Hamiltonian localization. In that limit, the Lagrange multipliers and antighosts become dynamically passive, which leads naturally to the appearance of the set of the delta - functionals necessary as to the localization. In contrast to the genuine relativistic gauge theories, now the gauge - fixing functions is intended to kill the dynamically active Lagrange multipliers to the gauge fixing itself, which looks a bit paradoxical, like an attempt of taking one from a bog by using the Münchhausen trick. However, that is actually the case. In the approach suggested, just the gauge - fixing function plays the role of an interpolation "parameter" switching on quantum fluctuations.

Further, as we have known that the whole field - antifield formalism [14, 15] is derived from the BRST-BFV quantization scheme [16, 17, 18, 19], it appears quite natural that there should be possible to describe the localization phenomenon just in the language of the field - antifield formalism, as well. We realize that program, by formulating a very specific version of the 
classical master - action. The action is a homogeneous linear function of the antifields, so that it vanishes at zero values of the ones. There is no term of the zero order in the antifields. The latter circumstance makes it possible for the unity matrix to stand for the "gauge generator". Then, we also introduce a very specific version to the gauge - fixing function, which is proportional to the extremal of the original action. As a coefficient of that proportionality, we allow for an invertible field-dependent metric. There also included the term proportional to the Nakanishi Lautrup fields, with the coefficient being the delocalization parameter. As a result, at the zero value of the delocalization parameter, we arrive at the functional path integral for the partition function, where the integration over the Nakanishi - Lautrup - fields yield the delta - function of the extremal of the original action, while the integrations over the ghosts and antighosts yield the corresponding Jacobian correct. On the other hand, if the delocalization parameter is not zero, there appears the Gaussian distribution of the classical extremal, instead of the delta - function. In fact, as the metric and the delocalization parameter do enter the functional path integral via the gauge - fixing only, the whole partition function is independent of them, due to the standard general arguments [13, 14, 15, 20, 21].

\section{Hamiltonian localization in the BRST-BFV formalism}

Let $S$ be an original Hamiltonian action of some nonsingular dynamical system,

$$
S=\int d t\left[\frac{1}{2} Z^{A} \omega_{A B} \dot{Z}^{B}-H(Z)\right]
$$

where $Z^{A}$ are phase variables, $\varepsilon\left(Z^{A}\right)=\varepsilon_{A}, \omega_{A B}$ is a constant invertible symplectic metric, $H(Z)$ is a non-degenerate original Hamiltonian. The action (2.1) yields the standard equations of motion,

$$
\dot{Z}^{A}=\left\{Z^{A}, H(Z)\right\}_{\omega}=\omega^{A B} \partial_{B} H(Z)
$$

Now, we would like to perform a localization to the equation of motion (2.2) via the following extended action $W$,

$$
W=\int d t\left[P_{A} \dot{Z}^{A}+\Pi_{A} \dot{\Lambda}^{A}+\overline{\mathcal{P}}_{A} \dot{C}^{A}+\bar{C}_{A} \dot{\mathcal{P}}^{A}-\mathcal{H}\right]
$$

in relativistic phase space of canonical pairs with the values assigned of the Grassmann parities, $\varepsilon$, and ghost number, gh, original phase variables:

$$
\left(P_{A}, Z^{A}\right), \quad \varepsilon\left(Z^{A}\right)=\varepsilon\left(P_{A}\right)=\varepsilon_{A}, \quad \operatorname{gh}\left(Z^{A}\right)=-\operatorname{gh}\left(P_{A}\right)=0
$$

dynamically active Lagrange multipliers:

$$
\left(\Pi_{A}, \Lambda^{A}\right), \quad \varepsilon\left(\Lambda^{A}\right)=\varepsilon\left(\Pi_{A}\right)=\varepsilon_{A}, \quad \operatorname{gh}\left(\Lambda^{A}\right)=-\operatorname{gh}\left(\Pi_{A}\right)=0
$$


ghost phase variables:

$$
\left(\overline{\mathcal{P}}_{A}, C^{A}\right), \quad \varepsilon\left(C^{A}\right)=\varepsilon\left(\overline{\mathcal{P}}_{A}\right)=\varepsilon_{A}+1, \quad \operatorname{gh}\left(C^{A}\right)=-\operatorname{gh}\left(\overline{\mathcal{P}}_{A}\right)=1
$$

antighost phase variables:

$$
\left(\bar{C}_{A}, \mathcal{P}^{A}\right), \quad \varepsilon\left(\mathcal{P}^{A}\right)=\varepsilon\left(\bar{C}_{A}\right)=\varepsilon_{A}+1, \quad \operatorname{gh}\left(\mathcal{P}^{A}\right)=-\operatorname{gh}\left(\bar{C}_{A}\right)=1 .
$$

The unitarizing extended Hamiltonian is given by

$$
\mathcal{H}=\{\Psi, \Omega\}
$$

where gauge Fermion $\Psi$ and BRST charge $\Omega$ are

$$
\begin{gathered}
\Psi=\overline{\mathcal{P}}_{A} \Lambda^{A}+\bar{C}_{A} \chi^{A}, \quad \varepsilon(\Psi)=1, \quad \operatorname{gh}(\Psi)=-1, \\
\Omega=H \overleftarrow{\partial_{A}} C^{A}+\Pi_{A} \mathcal{P}^{A}, \quad \varepsilon(\Omega)=1, \quad \operatorname{gh}(\Omega)=1, \quad\{\Omega, \Omega\}=0,
\end{gathered}
$$

where $\chi^{A}$ are just gauge - fixing functions, $\varepsilon\left(\chi^{A}\right)=\varepsilon_{A}, \operatorname{gh}\left(\chi^{A}\right)=0$, which may depend on all the phase variables, and $\partial_{A}$ is everywhere a partial $Z^{A}$ - derivative. The minimal form (2.8) of the unitarizing Hamiltonian $\mathcal{H}$ is directly related to the possibility to use actually the so-called quantum/derived antibrackets [22, 23, 24, 25] as to describe dynamical systems with first-class constraints (see [26] for details); see also [27, 28] for the modified description of the time evolution for general gauge systems.

By substituting (2.9), (2.10) into (2.8), we get the following explicit form of the unitarizing extended Hamiltonian,

$$
\mathcal{H}=H \overleftarrow{\partial_{A}} \Lambda^{A}+\Pi_{A} \chi^{A}+\bar{C}_{A}\left\{\chi^{A}, \Pi_{B} \mathcal{P}^{B}\right\}+\bar{C}_{A}\left\{\chi^{A}, H \overleftarrow{\partial_{B}} C^{B}\right\}+\overline{\mathcal{P}}_{A} \mathcal{P}^{A}
$$

All the Poisson brackets in (2.8) - (2.11) are defined with respect to the canonical pairs in (2.4) - (2.7). In terms of the extended action (2.3), the partition function reads

$$
\mathcal{Z}=\int D \Gamma \exp \left\{\frac{i}{\hbar} W\right\}
$$

where $D \Gamma$ is a trivial integration measure over all the phase variables (2.4) - (2.7).

Due to the standard general arguments based on the Poisson - bracket nilpotency of $\Omega$ in (2.10), the partition function (2.12) is independent of the gauge Fermion $\Psi$, and thereby of the gauge - fixing functions $\chi^{A}[11]$. On the other hand, for general $\chi^{A}$, of course, the functional path integral (2.12) is not localized to a solution to the classical equation of motion (2.2), with the Jacobian of the corresponding delta functional being compensated exactly. We will show however that there exists such a choice for $\chi^{A}$ that the required localization is the case in the unitary limit of the path integral in (2.12). So, let us define the unitary limit in the usual way 
$[11,12,13,14,15,16]$. As the partition function (2.12) is independent of $\chi^{A}$, let us formally rescale the latter

$$
\chi^{A} \rightarrow \alpha^{-1} \chi^{A}
$$

where $\alpha$ is a parameter. Then, let us make the following unimodular change of integration variables in (2.12),

$$
\Pi_{A} \quad \rightarrow \alpha \Pi_{A}, \quad \bar{C}_{A} \rightarrow \alpha \bar{C}_{A}
$$

Now, let us go to the limit $\alpha \rightarrow 0$. It is easy to see that the structure of the Hamiltonian (2.11) is preserved under the limit. The only result of the unitary limit in the extended action $W$ is that the second and fourth kinetic terms in the integrand in (2.3) become absent, so that the Lagrange multipliers (2.5) and antighosts (2.7) become dynamically - passive variables. It is now the case to choose a special gauge - fixing,

$$
\chi^{A}=\Lambda^{A}-P_{B} \omega^{B A} .
$$

In the latter case, the $\Pi_{A}$ - integration yields the delta functional $\delta[\Lambda-P \omega]$, while the $\mathcal{P}^{A}-$ integration yields the delta-functional $\delta[\overline{\mathcal{P}}+\bar{C}]$. By integrating out these delta - functionals, we have to substitute

$$
\Lambda^{A}=P_{B} \omega^{B A}, \quad \overline{\mathcal{P}}_{A}=-\bar{C}_{A},
$$

so that the final action becomes

$$
W_{\text {final }}=\int d t\left[P_{A}\left(\dot{Z}^{A}-\omega^{A B} \partial_{B} H\right)-\bar{C}_{A}\left(\dot{C}^{A}-\omega^{A C} \overrightarrow{\partial_{C}} H \overleftarrow{\partial_{B}} C^{B}\right)\right]
$$

Here, the $P_{A}$ - integration yields the delta-functional

$$
\delta\left[\dot{Z}-\{Z, H\}_{\omega}\right]
$$

while the $\bar{C}_{A}$ and $C^{B}$ - integrations yield exactly the Jacobian of the argument in (2.18) with respect to $Z$. Thus, we have reproduced exactly the result of Hamiltonian localization.

Now, it is worth to compare the final action (2.17) to the known approach based on the use of the two extra time Fermionic variables $\theta^{a}, a=1,2$, where one proceeds with the two-parametric superfield action of the form [9, 29]

$$
W^{\prime}=\int d t d^{2} \theta\left[\frac{1}{2} Z^{A} \omega_{A B} \dot{Z}^{B}-H(Z)\right], \quad \omega_{A B}=\operatorname{const}(Z),
$$

where

$$
Z^{A}\left(t, \theta^{1}, \theta^{2}\right)=Z_{0}^{A}(t)+\theta^{a} Z_{a}^{A}(t)+\delta^{2}(\theta) Z_{3}^{A}(t)
$$


is the component expansion of the two-parametric superfield,

$$
\begin{aligned}
& d^{2} \theta=d \theta^{1} d \theta^{2}, \quad \delta^{2}(\theta)=\frac{1}{2} \theta^{a} \varepsilon_{a b} \theta^{b} \\
& \int d^{2} \theta \delta^{2}(\theta)=1, \quad \int d^{2} \theta \theta^{a} \theta^{b}=-\varepsilon^{a b},
\end{aligned}
$$

while (2.21), (2.22) represent the two-parametric integration measure, delta-function, and the normalization properties. Due to (2.20) - (2.22), the action (2.19) rewrites in the component form

$$
W^{\prime}=\int d t\left[Z_{3}^{A}\left(\omega_{A B} \dot{Z}_{0}^{B}-\partial_{A} H\left(Z_{0}\right)\right)+\frac{1}{2} \varepsilon^{a b}\left(Z_{a}^{A} \omega_{A B} \dot{Z}_{b}^{B}-Z_{a}^{A} \overrightarrow{\partial_{A}} H \overleftarrow{\partial_{B}} Z_{b}^{B}\right)(-1)^{\varepsilon_{B}}\right] .
$$

It follows when comparing (2.17) to (2.23) that the variables do identify as

$$
Z_{3}^{A} \omega_{A B}=P_{B}, \quad Z_{1}^{A} \omega_{A B}=\bar{C}_{B}, \quad-Z_{2}^{B}(-1)^{\varepsilon_{B}}=C^{B} .
$$

Notice that the explicitly $N=2$ supersymmetric form of the action (2.19) reads [30]

$$
W^{\prime}=\int d t d^{2} \theta\left[-\frac{1}{4} D_{a} Z^{A} \omega_{A B} g^{a b} D_{b} Z^{B}(-1)^{\varepsilon_{B}}-H(Z)\right]
$$

where covariant superderivatives are given by

$$
D_{a}=\frac{\partial}{\partial \theta^{a}}+g_{a b} \theta^{b} \frac{\partial}{\partial t}
$$

with

$$
g_{a b}=g_{b a}=\text { const }, \quad \varepsilon\left(g_{a b}\right)=0,
$$

being an invertible symmetric constant metric, and $g^{a b}$ being an inverse to the latter. Due to the supercommutation relations

$$
\left[D_{a}, D_{b}\right]=2 g_{a b} \frac{\partial}{\partial t}
$$

the explicitly $N=2$ supersymmetric action (2.25) coincides exactly with the one (2.19) after integration by part.

\section{Some possible generalizations}

We have demonstrated an interesting possibility of the Hamiltonian generalized BRST-BFV formalism [11, 13], being a very powerful quantization method of arbitrary dynamical systems with constraints, to describe a phenomena of the Hamiltonian localization of classical mechanics. To achieve this result it was necessary to change the standard view on gauge fixing functions of the BRST-BFV approach allowing them to depend on the variables of the auxiliary sectors rather than on the initial phase space variables. Then, using the fact of the gauge independence 
of the partition function constructed by the rules of the BRST-BFV method, an application of the unitary limit procedure allowed one to make the Lagrange multipliers and the antighost variables dynamically - passive. As the last step in our proof of the Hamiltonian localization, it was a special choice of the gauge fixing functions in the BRST-BFV formalism.

Now, we would like to make a backward step as to explain our understanding of what is the meaning of minimal Hamiltonian delocalization. Let us modify slightly the unitary gauge (2.15),

$$
\chi^{A}=\Lambda^{A}-P_{A} \omega^{B A}+\frac{1}{2} g^{A B} \Pi_{B}
$$

where $g^{A B}$ is an invertible matrix with the property of dual antisymmetry,

$$
g^{A B}=-g^{B A}(-1)^{\left(\varepsilon_{A}+1\right)\left(\varepsilon_{B}+1\right)} .
$$

Write down the gauge fixing term in (2.11), with the gauge (3.1) substituted for (2.15),

$$
\Pi_{A} \chi^{A}=\Pi_{A}\left(\Lambda^{A}-P_{B} \omega^{B A}\right)+\frac{1}{2} \Pi_{A} g^{A B} \Pi_{B} .
$$

With nonzero invertible $g^{A B}$, the Lagrange multipliers $\Pi_{A}$ enter quadratically (3.3), so that in the functional path integral, in the unitary limit, the $\Pi_{A}$ integration yields a Gaussian distribution of the unitary gauges (2.15), instead of the delta - functional of (2.15). That is actually what we mean when saying about the minimal Hamiltonian delocalization.

Finally, let us discuss another important aspect of the construction. So far, we assumed the original symplectic metric $\omega_{A B}$ as being constant. Now, let us allow the metric be dependent of the original phase variables $Z^{A}$, although satisfying the Jacobi relation,

$$
\omega^{A B} \overleftarrow{\partial_{D}} \omega^{D C}(-1)^{\varepsilon_{A} \varepsilon_{C}}+\operatorname{cycle}(A, B, C)=0
$$

In order to have the original equations of motion be derivable from the action of the form (2.1), we have to modify the latter as follows (for details see [21])

$$
\frac{1}{2} \omega_{A B} \quad \rightarrow \quad \bar{\omega}_{A B}=(N+2)^{-1} \omega_{A B}, \quad N=Z^{A} \partial_{A} .
$$

If one looks at the formula (2.17), it becomes clear that the $\bar{C}_{A}, C^{B}$ integrations yield the correct Jacobian only if the operator $\overleftarrow{\partial}_{B}$ applies both to $H$ and to $\omega^{A C}$. On the other hand, our above arguments are only capable to justify applying the operator $\overleftarrow{\partial}_{B}$ to the $H$ only. To resolve that problem, we should modify the gauge Fermion $\Psi(2.9),(2.15)$ as

$$
\Psi \rightarrow \Psi+\frac{1}{2}(-1)^{\varepsilon_{D}} \overline{\mathcal{P}}_{D} \overline{\mathcal{P}}_{A} \omega^{A D} \overleftarrow{\partial_{B}} C^{B}
$$

Then the unitarizing Hamiltonian $\mathcal{H}(2.8)$, (2.11) modifies as

$$
\mathcal{H} \rightarrow \mathcal{H}+\overline{\mathcal{P}}_{A} \omega^{A D} \overleftarrow{\partial_{B}} \overrightarrow{\partial_{D}} H C^{B}(-1)^{\varepsilon_{B} \varepsilon_{D}}
$$


which results exactly in the formula (2.17) where in the right-hand side, in the integrand, in the second term, the left derivative $\overleftarrow{\partial_{B}}$ applies both to $H$ and to $\omega^{A C}$. Thereby, the $\bar{C}, C$ integrations yield exactly the Jacobian of the argument of the delta function yielded via the $P$ integration in the first term in the integrand.

\section{Lagrangian localization in field-antifield formalism}

As we have known that the whole field-antifield formalism is derived from the Hamiltonian BRST- BFV quantization scheme [11, 13], it appears quite natural to expect that there should be possible to describe the localization phenomenon just in the language of the field - antifield formalism, as well. Let us begin with determining the set of anticanonical pairs of the fieldantifield phase variables, with the values assigned of the Grassmann parity, $\varepsilon$, and ghost number, gh. For general field variables, $\Phi^{A}$, and their antifields, $\Phi_{A}^{*}$, we have

$$
\varepsilon\left(\Phi^{A}\right)=\varepsilon_{A}, \quad \varepsilon\left(\Phi_{A}^{*}\right)=\varepsilon_{A}+1, \quad \operatorname{gh}\left(\Phi^{A}\right)+\operatorname{gh}\left(\Phi_{A}^{*}\right)=-1
$$

For any functions $F, G$ on the antisymplectic phase space, the antibracket is defined by

$$
(F, G)=F\left(\overleftarrow{\partial_{A}} \vec{\partial}_{*}^{A}-\overleftarrow{\partial}{ }_{*}^{A} \overrightarrow{\partial_{A}}\right) G
$$

where $\partial_{A}$ and $\partial_{*}^{A}$ is the $\Phi^{A}$ and $\Phi_{A}^{*}$ - derivative, respectively.

Now, we proceed with the following specific set of anticanonical pairs.

1. Original phase variables: $\phi^{i}, \phi_{i}^{*}$,

$$
\varepsilon\left(\phi^{i}\right)=\varepsilon_{i}, \quad \varepsilon\left(\phi_{i}^{*}\right)=\varepsilon_{i}+1, \quad \operatorname{gh}\left(\phi^{i}\right)=0, \quad \operatorname{gh}\left(\phi_{i}^{*}\right)=-1
$$

2. Nakanishi - Lautrup phase variables: $B_{i}, B^{* i}$,

$$
\varepsilon\left(B_{i}\right)=\varepsilon_{i}, \quad \varepsilon\left(B^{* i}\right)=\varepsilon_{i}+1, \quad \operatorname{gh}\left(B_{i}\right)=0, \quad \operatorname{gh}\left(B^{* i}\right)=-1 ;
$$

3. Ghost phase variables: $C^{i}, C_{i}^{*}$,

$$
\varepsilon\left(C^{i}\right)=\varepsilon_{i}+1, \quad \varepsilon\left(C_{i}^{*}\right)=\varepsilon_{i}, \quad \operatorname{gh}\left(C^{i}\right)=1, \quad \operatorname{gh}\left(C_{i}^{*}\right)=-2 ;
$$

4. Antighost phase variables: $\bar{C}_{i}, \bar{C}^{* i}$,

$$
\varepsilon\left(\bar{C}_{i}\right)=\varepsilon_{i}+1, \quad \varepsilon\left(\bar{C}^{* i}\right)=\varepsilon_{i}, \quad \operatorname{gh}\left(\bar{C}_{i}\right)=-1, \quad \operatorname{gh}\left(\bar{C}^{* i}\right)=0 .
$$

Now, let $\mathcal{S}(\phi)$ be an original action whose Hessian,

$$
H_{i k}=\overrightarrow{\partial_{i}} \mathcal{S} \overleftarrow{\partial_{k}}
$$


is invertible at the extremals. Here $\partial_{i}$ is the $\phi^{i}$ - derivative. Next, we define the following classical master - action,

$$
S=\phi_{i}^{*} C^{i}+B_{i} \bar{C}^{* i}, \quad \varepsilon(S)=0, \quad \operatorname{gh}(S)=0, \quad(S, S)=0
$$

The gauge - fixing Fermion has the form,

$$
\Psi=\bar{C}_{i} \chi^{i}, \quad \varepsilon(\Psi)=1, \quad \operatorname{gh}(\Psi)=-1,
$$

where just the gauge - fixing function is defined by

$$
\chi^{i}=g^{i k}\left(\partial_{k} \mathcal{S}+\frac{1}{2} \kappa B_{k}\right), \quad \varepsilon\left(\chi^{i}\right)=\varepsilon_{i}, \quad \operatorname{gh}\left(\chi^{i}\right)=0,
$$

$\kappa$ is the delocalization parameter. Metric $g^{i k}$ is invertible and allowed to depend on $\phi^{i}$, it has the dual anti-symmetry property,

$$
g^{i k}=-g^{k i}(-1)^{\left(\varepsilon_{i}+1\right)\left(\varepsilon_{k}+1\right)} .
$$

The quantum partition function reads

$$
\mathcal{Z}=\int D \phi D B D C D \bar{C} \exp \left\{\frac{i}{\hbar} S_{\Psi}\right\},
$$

where the gauge - fixed master - action is

$$
S_{\Psi}=\left.S\right|_{\phi^{*}=\partial \Psi, \bar{C}^{*}=\chi}=\bar{C}_{i} g^{i k}\left(\overrightarrow{\partial_{k}} \mathcal{S}+\frac{1}{2} \kappa B_{k}\right) \overleftarrow{\partial_{j}} C^{j}+B_{i} g^{i k}\left(\overrightarrow{\partial_{k}} \mathcal{S}+\frac{1}{2} \kappa B_{k}\right)
$$

As the metric $g^{i k}$ and delocalization parameter $\kappa$ do enter via the gauge fixing (4.10) only, the partition function is independent of them, due to the standard general arguments [13, 14, 15, 20, 21]. Formally, for $\kappa=0$, the $B^{i}$ - integration yields the delta - function of the extremal of the action $\mathcal{S}$, while the $\bar{C}_{i}, C^{k}$ - integrations yield the corresponding Jacobian correct.

The solution chosen (4.8) for the classical master - action is a very specific one. It is a homogeneous linear function of the antifields, so that it vanishes at zero values of the ones. There is no term in (4.8) of zero order in the antifelds. The latter circumstance makes it possible to choose the unity matrix to stand for the "gauge generator".

\section{Acknowledgments}

I. A. Batalin would like to thank Klaus Bering of Masaryk University for interesting discussions. The work of I. A. Batalin is supported in part by the RFBR grants 14-01-00489 and 14-0201171. The work of P. M. Lavrov is supported by the Ministry of Education and Science of Russian Federation, grant project 2014/387/122. 


\section{References}

[1] B. S. De Witt, Dynamical theory of groups and fields, (Gordon and Breach, NY 1965).

[2] S. Weinberg, The Quantum Theory of Fields, Vol. II (Cambridge University Press, Cambridge, 1996).

[3] M. Henneaux and C. Teitelboim, Quantization of gauge systems , (Princeton University Press, 1992).

[4] C. Becchi, A. Rouet and R. Stora, The abelian Higgs Kibble Model, unitarity of the Soperator, Phys. Lett. B52 (1974) 344.

[5] I. V. Tyutin, Gauge invariance in field theory and statistical physics in operator formalism, Lebedev Institute preprint No. 39 (1975), arXiv:0812.0580 [hep-th].

[6] E. Gozzi, Hidden BRS invariance in Classical Mechanics Phys. Lett. B201 (1988) 525.

[7] E. Gozzi, M. Reuter and W. D. Thacker, Hidden BRS invariance in Classical Mechanics. II, Phys. Rev. D40 (1989) 3363.

[8] E. Gozzi, M. Reuter and W. D. Thacker, Symmetries of the classical path integral on a generalized phase space manifold, Phys. Rev. D46 (1992) 757.

[9] E. Deotto and E. Gozzi, On the 'universal' $N=2$ supersymmetry of classical mechanics, Int. J. Mod. Phys. A16 (2001) 2709.

[10] E. Cattaruzza, E. Gozzi and A. Francisco Neto, Least-action principle and path-integral for classical mechanics, Phys. Rev. D87 (2013) 6, 067501.

[11] E. S. Fradkin and G. A. Vilkovisky, Quantization of relativistic systems with constraints, Phys. Lett. B55 (1975) 224.

[12] E. S. Fradkin and G. A. Vilkovisky, Quantization of Relativistic Systems with Constraints: Equivalence of Canonical and Covariant Formalisms in Quantum Theory of Gravitational Field, Preprint CERN-TH-2332, 1977, 53 pp.

[13] I. A. Batalin and G. A. Vilkovisky, Relativistic S-matrix of dynamical systems with boson and fermion constraints, Phys. Lett. B69 (1977) 309.

[14] I. A. Batalin and G. A. Vilkovisky, Gauge algebra and quantization, Phys. Lett. B102 (1981) 27.

[15] I. A. Batalin and G. A. Vilkovisky, Quantization of gauge theories with linearly dependent generators, Phys. Rev. D28 (1983) 2567.

[16] I. A. Batalin and E. S. Fradkin, Operatorial quantizaion of dynamical systems subject to constraints. A Further study of the construction, Annales Inst. Henri Poincare Phys. Theor. 49 (1988) 145.

[17] G.V. Grigorian, R.P. Grigorian and I.V. Tyutin, Equivalence of Lagrangian and Hamiltonian BRST quantizations: Systems with first class constraints, Sov. J. Nucl. Phys. 53 (1991) 1058. 
[18] G.V. Grigorian, R.P. Grigorian and I.V. Tyutin, Equivalence of Lagrangian and Hamiltonian BRST quantizations: The General case, Nucl. Phys. B379 (1992) 304.

[19] I. A. Batalin and I. V. Tyutin, On the perturbative equivalence between the Hamiltonian and Lagrangian quantizations, Int. J. Mod. Phys. A11 (1996) 1353.

[20] R. E. Kallosh and I. V. Tyutin, The equivalence theorem and gauge invariance in renormalizable theories, Sov. J. Nucl. Phys. 17 (1973) 98.

[21] I. A. Batalin and E. S. Fradkin, Formal path integral for theories with noncanonical commutation relations, Mod. Phys. Lett. A4 (1989) 1001.

[22] Y. Kosmann-Schwarzbach, From Poisson algebras for Gerstenhaber algebras, Ann. Inst. Fourier (Grenoble) 46 (1996) 1241.

[23] Y. Kosmann-Schwarzbach, Derived brackets, Lett. Math. Phys. 69 (2004) 61.

[24] I. Batalin and R. Marnelius, Quantum antibrackets, Phys. Lett. B434 (1998) 312.

[25] I. Batalin and R. Marnelius, General quantum antibrackets, Theor. Math. Phys. 120 (1999) 1115 .

[26] I. A. Batalin and P. M. Lavrov, Superfield Hamiltonian quantization in terms of quantum antibracket, Int. J. Mod. Phys. A 31 (2016) 1650054.

[27] R. Marnelius, Time evolution in general gauge theories, Talk at the International Workshop "New Non Perturbative Methods and Quantization on the Light Cone", Les Houches, France, Feb.24-March 7, 1997.

[28] R. Marnelius, Time evolution in general gauge theories on inner product spaces, Nucl. Phys. B494 (1997) 346.

[29] I. A. Batalin and K. Bering, Hamiltonian superfield formalism with $N$ supercharges, Nucl. Phys. B700 (2004) 439.

[30] C. Hull, The geometry of supersymmetric quantum mechanics, arXiv:hep-th/9910028. 